\title{
A Two Parameter Chromatic Symmetric Function
}

\author{
Ellison-Anne Williams \\ kingpuck20@yahoo.com
}

\author{
Submitted: Jun 17, 2004; Accepted: Jan 29, 2007; Published: Feb 12, 2007 \\ Mathematics Subject Classification: 05C88
}

\begin{abstract}
We introduce and develop a two-parameter chromatic symmetric function for a simple graph $G$ over the field of rational functions in $q$ and $t, \mathbb{Q}(q, t)$. We derive its expansion in terms of the monomial symmetric functions, $m_{\lambda}$, and present various correlation properties which exist between the two-parameter chromatic symmetric function and its corresponding graph.

Additionally, for the complete graph $G$ of order $n$, its corresponding two parameter chromatic symmetric function is the Macdonald polynomial $Q_{(n)}$. Using this, we develop graphical analogues for the expansion formulas of the two-row Macdonald polynomials and the two-row Jack symmetric functions.

Finally, we introduce the "complement" of this new function and explore some of its properties.
\end{abstract}

\section{Preliminaries.}

We briefly define some of the basic concepts used in the development of our two parameter chromatic symmetric function. In general, our notation will be consistent with that of [1].

Let $G$ be a finite, simple graph; $G$ has no multiple edges or loops. Denote the edge set of $G$ by $E(G)$ and the vertex set of $G$ by $V(G)$. The order of the graph $G$, denoted $o(G)$, is the size of its vertex set $V(G)$ and the size of the graph $G$, denoted $s(G)$, is equal to the number of edges in $E(G)$. A subgraph of $G, G^{\prime}$, is a graph whose vertex set and edge set are contained in those of $G$. For a subset $V^{\prime}(G) \subseteq V(G)$, the subgraph induced by $V^{\prime}(G), G_{I}$, is the subgraph of $G$ which contains all edges in $E(G)$ which connect any two vertices in $V^{\prime}(G)$.

For the graph $G$, denote the edge of $E(G)$ which joins the vertices $v_{i}, v_{j} \in V(G)$ by $v_{i} v_{j}$; we say that $v_{i}$ and $v_{j}$ are the endvertices of the edge $v_{i} v_{j}$. A walk in $G$ is a sequence of vertices and edges, $v_{1}, v_{1} v_{2}, \ldots, v_{l-1} v_{l}, v_{l}$, denoted $v_{1} \ldots v_{l}$; the length of this walk is $l$. A path is a walk with distinct vertices and a trail is a walk with distinct edges. A trail whose endvertices are equal, $v_{1}=v_{l}$, is called a circuit. A walk of length $\geq 3$ whose vertices are all distinct, except for coinciding endvertices, is called a cycle. The graph $G$ 
is said to be connected if for every pair of vertices $\left\{v_{i}, v_{j}\right\} \in V(G)$, there is a path from $v_{i}$ to $v_{j}$. A tree is a connected, acyclic graph.

Let $V(G)=\left\{v_{1}, \ldots, v_{n}\right\}$. Denote the number of edges emminating from the vertex $v_{i} \in V(G)$ by $d\left(v_{i}\right)$, the degree of the vertex $v_{i}$. The degree sequence of $G$, denoted by $\operatorname{deg}(G)$, is a weakly decreasing sequence (or partition) of nonnegative integers, $\operatorname{deg}(G)=$ $\left(d_{1}, \ldots, d_{n}\right)$, such that the length of $\operatorname{deg}(G)$ is equal to $|V(G)|$ and $\left(d_{1}, \ldots, d_{n}\right)$ represents the degrees of the vertices of $V(G)$, arranged in decreasing order. Since each edge of $G$ has two endvertices, it follows that $\sum_{i=1}^{n} d_{i}=2 s(G)$; thus, $\operatorname{deg}(G) \vdash 2 s(G)$.

A coloring of the graph $G$ is a function $k: V(G) \rightarrow \mathbb{N}$. The coloring $k$ is said to be proper if $k\left(v_{i}\right) \neq k\left(v_{j}\right)$ whenever $v_{i} v_{j} \in E(G)$.

Additionally, we will use the following consistent with [2].

$$
\begin{gathered}
(a ; q)_{0}=1 \\
(a ; q)_{n}=\prod_{i=0}^{n-1}\left(1-a q^{i}\right) \\
(a ; q)_{n}=\frac{(a ; q)_{\infty}}{\left(a q^{n} ; q\right)_{\infty}} \\
\left(a_{1}, \ldots, a_{m} ; q\right)_{n}=\left(a_{1} ; q\right)_{n} \cdots\left(a_{m} ; q\right)_{n} \\
(a ; q)=(a ; q)_{1}
\end{gathered}
$$

\section{A Two-Parameter Chromatic Symmetric Function.}

Let $G$ be a simple graph with vertex set $V(G)=\left\{v_{1}, \ldots, v_{n}\right\}$ and let $k: V(G) \rightarrow \mathbb{N}$ be a coloring from the set of vertices of the graph $G$ into $\mathbb{N}=\{1,2, \ldots\}$. An edge $v_{i} v_{j} \in E(G)$ is colored c by $k$ if $k\left(v_{i}\right)=k\left(v_{j}\right)=c$. Denote $m_{i}(k)$ to be the number of monochromatic edges of $G$ which are colored $i \in \mathbb{N}$ with respect to the coloring $k$. Denote $R(k)$ to be the range of the coloring $k$.

For $i \in \mathbb{N}$, as in [6], set

$$
V_{i}=\left|\left\{v_{j} \in V(G): k\left(v_{j}\right)=i\right\}\right|
$$

i.e. the number of vertices of $V(G)$ colored $i$ by $k$. For $i \in R(k)$, define

$$
m_{i}= \begin{cases}\left(m_{i}(k)+1\right) & \text { if }\left(m_{i}(k)+1\right) \leq V_{i} \\ V_{i} & \text { otherwise. }\end{cases}
$$

Let $x=\left\{x_{1}, x_{2}, \ldots\right\}$ be a set of commuting indeterminates. For the coloring $k$ : $V(G) \rightarrow \mathbb{N}$, set

$$
x^{k}=\prod_{i=1}^{n} x_{k\left(v_{i}\right)}
$$

for $v_{i} \in V(G)$. 
Definition 2.1. For a simple graph $G, o(G)=n$,

$$
Y_{G}(x ; q, t)=\sum_{k}\left(\begin{array}{c}
n \\
V_{1}, V_{2}, \ldots
\end{array}\right)^{-1}\left(\prod_{i \in R(k)} \frac{(t ; q)_{m_{i}}}{(q ; q)_{m_{i}}}\right) x^{k}
$$

where $k$ ranges over all colorings of $G$.

It follows from Definition 2.1 and $(3)$ that $Y_{G}(x ; q, t)$ is a symmetric function of degree $n$.

Remark 2.1. The papers [6] and [7], by Richard Stanley, served as inspiration for this work. Note however, that his chromatic symmetric function described is [6] and [7] and the present two-parameter chromatic symmetric function are entirely different. Some of the prominent differences include, for example, that the function in this paper is a two-parameter symmetric function in $q$ and $t$ and that the colorings considered here are not necessarily proper. Even if we set $q=\frac{1}{t}$ to kill the terms corresponding to colorings that are not proper, the remaining coefficients are different from Stanley's. See [6] and [7] for further details.

Definition 2.2. Let $\lambda=\left(\lambda_{1}, \ldots, \lambda_{n}\right)$ be a partition and $G$ be a simple graph.

A general distinct coloring is a coloring of $G, k_{\lambda}^{m}: V(G) \rightarrow \mathbb{N}$, which sends $\lambda_{i}$-many vertices to one color and $\lambda_{j}$-many vertices to another color, for all $i \neq j$.

The basic coloring of $G$ of type $\lambda, k_{\lambda}: V(G) \rightarrow \mathbb{N}$, is the set of all general distinct colorings $\left\{k_{\lambda}^{m}\right\}$ of the graph $G$.

Remark 2.2. Note that for $k_{\lambda}=\left\{k_{\lambda}^{m}\right\}$, each general distinct coloring $k_{\lambda}^{m}: V(G) \rightarrow \mathbb{N}$ corresponds to a unique, ordered grouping of the vertices of $V(G)$ into disjoint subsets of size $\lambda_{i}, 1 \leq i \leq n$.

In other words, the map $k_{\lambda}^{m}$ is a general distinct coloring if it partitions $V(G)$ into disjoint subsets of size $\lambda_{1}, \lambda_{2}, \ldots, \lambda_{n}$ such that the vertices in each subset are all mapped to the same color and such that the vertices in distinct subsets are mapped to distinct colors by $k_{\lambda}^{m}$.

Additionally, for $o(G)=d$, there are $\left(\begin{array}{c}d \\ \lambda_{1}, \ldots, \lambda_{n}\end{array}\right)$-many general distinct colorings, $k_{\lambda}^{m}$, within $k_{\lambda} ;\left|k_{\lambda}\right|=\left(\begin{array}{c}d \\ \lambda_{1}, \ldots, \lambda_{n}\end{array}\right)$.

Example 2.1. Let $\lambda=(3,2,1,1)$ and $V(G)=\left\{v_{1}, \ldots, v_{7}\right\}$. Let $\{j\}$ denote a subset of vertices of $V(G)$ of size $j$. The basic coloring of $G$ of type $\lambda=(3,2,1,1)$ includes all general distinct colorings $k_{\lambda}^{m}: V(G) \rightarrow \mathbb{N}$ such that $k_{\lambda}^{m}(\{3\}) \neq k_{\lambda}^{m}(\{2\}) \neq k_{\lambda}^{m}(\{1\}) \neq k_{\lambda}^{m}(\{1\})$; each $m$ corresponds to a specific ordered grouping, $(\{3\},\{2\},\{1\},\{1\})$, of disjoint $j$ element subsets of $V(G), j \in\{1,2,3,3\}$. Note that $\left|k_{\lambda}\right|=\left(\begin{array}{c}7 \\ 3,2,1,1\end{array}\right)=420$, the number of general distinct colorings, $k_{\lambda}^{m}$, included in the basic coloring $k_{\lambda}$ :

$$
k_{\lambda}=\left\{\left(\left\{v_{1}, v_{2}, v_{3}\right\},\left\{v_{4}, v_{5}\right\},\left\{v_{6}\right\},\left\{v_{7}\right\}\right),\left(\left\{v_{1}, v_{2}, v_{3}\right\},\left\{v_{4}, v_{5}\right\},\left\{v_{7}\right\},\left\{v_{6}\right\}\right), \ldots\right\} .
$$


Example 2.2. Consider the simple graph $G$ such that $V(G)=\left\{v_{1}, v_{2}, v_{3}, v_{4}\right\}$ and $E(G)=\left\{v_{1} v_{2}, v_{1} v_{3}, v_{2} v_{3}, v_{2} v_{4}\right\}$.

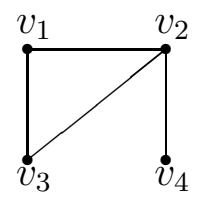

There are five possible basic colorings $k: V(G) \rightarrow \mathbb{N}:\left(1\right.$.) the coloring of type $\lambda=\left(1^{4}\right)$ sending each vertex to a different color, (2.) the coloring of type $\lambda=(4)$ sending all vertices to the same color, (3.) the coloring of type $\lambda=(3,1)$ which sends three vertices to the same color and the remaining one to a different color, (4.) the coloring of type $\lambda=(2,1,1)$ sending two vertices to the same color and sending the remaining two vertices to two other distinct colors, and (5.) the coloring of type $\lambda=(2,2)$ which sends two vertices to the same color and the remaining two vertices to the same color (distinct from the first).

Restrict the number of variables to four such that $x=\left\{x_{1}, x_{2}, x_{3}, x_{4}\right\}$. Therefore, the range of $k$ becomes $\{1,2,3,4\}, k: V(G) \rightarrow\{1,2,3,4\}$. We will compute $Y_{G}(x ; q, t)$ via computing the function of each of the five basic colorings.

Within the first basic coloring, there are $\left(\begin{array}{c}4 \\ 1,1,1,1\end{array}\right)=4$ ! general distinct colorings, each with $m_{i}=1$ for all $i \in\{1,2,3,4\}$ :

$$
\frac{(t ; q)^{4}}{(q ; q)^{4}} x_{1} x_{2} x_{3} x_{4}
$$

For the second basic coloring, there is $\left(\begin{array}{l}4 \\ 4\end{array}\right)=1$ general distinct coloring and four specific colorings. Since the range of the coloring is restricted to $\{1,2,3,4\}$, each of these gives $m_{i}=4$ for all $i \in\{1,2,3,4\}$ :

$$
\frac{(t ; q)_{4}}{(q ; q)_{4}}\left(x_{1}^{4}+x_{2}^{4}+x_{3}^{4}+x_{4}^{4}\right)
$$

There are $\left(\begin{array}{c}4 \\ 3,1\end{array}\right)=4$ general distinct colorings within the third basic coloring. For $\{3\}$, three of these give $m_{i}=3$ and one gives $m_{i}=2, k_{\lambda}^{m}=\left(\left\{v_{1}, v_{3}, v_{4}\right\},\left\{v_{2}\right\}\right)$ for all $i \in\{1,2,3,4\}$ :

$$
\frac{3}{4} \frac{(t ; q)_{3}(t ; q)}{(q ; q)_{3}(q ; q)}\left(x_{1}^{3} x_{2}+x_{2}^{3} x_{3}+\ldots\right)+\frac{1}{4} \frac{(t ; q)_{2}(t ; q)}{(q ; q)_{2}(q ; q)}\left(x_{1}^{3} x_{2}+x_{2}^{3} x_{3}+\ldots\right) .
$$

Within the fourth basic coloring, there are $\left(\begin{array}{c}4 \\ 2,1,1\end{array}\right)=12$ general distinct colorings. For the subset $\{2\}$, eight of these give $m_{i}=2$ and four give $m_{i}=1$ for all $i \in\{1,2,3,4\}$. Thus, we have:

$$
\frac{2}{3} \frac{(t ; q)_{2}(t ; q)(t ; q)}{(q ; q)_{2}(q ; q)(q ; q)}\left(x_{1}^{2} x_{2} x_{3}+x_{1} x_{2}^{2} x_{3}+\ldots\right)+\frac{1}{3} \frac{(t ; q)^{3}}{(q ; q)^{3}}\left(x_{1}^{2} x_{2} x_{3}+x_{2}^{2} x_{1} x_{3}+\ldots\right) .
$$


Lastly, within the fifth basic coloring, there are $\left(\begin{array}{c}4 \\ 2,2\end{array}\right)=6$ general distinct colorings, yielding:

$$
\frac{2}{3} \frac{(t ; q)_{2}(t ; q)}{(q ; q)_{2}(q ; q)}\left(x_{1}^{2} x_{2}^{2}+x_{2}^{2} x_{3}^{2}+\ldots\right)+\frac{1}{3} \frac{(t ; q)_{2}(t ; q)_{2}}{(q ; q)_{2}(q ; q)_{2}}\left(x_{1}^{2} x_{2}^{2}+x_{2}^{2} x_{3}^{2}+\ldots\right) .
$$

Thus,

$$
\begin{aligned}
& Y_{G}(x ; q, t)=\frac{(t ; q)^{4}}{(q ; q)^{4}} x_{1} x_{2} x_{3} x_{4}+\frac{(t ; q)_{4}}{(q ; q)_{4}}\left(x_{1}^{4}+x_{2}^{4}+x_{3}^{4}+x_{4}^{4}\right) \\
& +\left(\frac{3}{4} \frac{(t ; q)_{3}(t ; q)}{(q ; q)_{3}(q ; q)}+\frac{1}{4} \frac{(t ; q)_{2}(t ; q)}{(q ; q)_{2}(q ; q)}\right)\left(x_{1}^{3} x_{2}+x_{2}^{3} x_{3}+\ldots\right) \\
& \quad+\left(\frac{2}{3} \frac{(t ; q)_{2}(t ; q)(t ; q)}{(q ; q)_{2}(q ; q)(q ; q)}+\frac{1}{3} \frac{(t ; q)^{3}}{(q ; q)^{3}}\right)\left(x_{1}^{2} x_{2} x_{3}+x_{1} x_{2}^{2} x_{3}+\ldots\right) \\
& +\left(\frac{2}{3} \frac{(t ; q)_{2}(t ; q)}{(q ; q)_{2}(q ; q)}+\frac{1}{3} \frac{(t ; q)_{2}(t ; q)_{2}}{(q ; q)_{2}(q ; q)_{2}}\right)\left(x_{1}^{2} x_{2}^{2}+x_{2}^{2} x_{3}^{2}+\ldots\right) .
\end{aligned}
$$

As in [5], a set partition $P$ of the set $S$ is a collection of disjoint subsets $\left\{S_{1}, \ldots, S_{r}\right\}$ whose union is $S$. The set partition $P$ has type $\mu$ if $\mu=\left(\left|S_{1}\right|, \ldots,\left|S_{r}\right|\right)$ where $\left|S_{1}\right| \geq$ $\ldots \geq\left|S_{r}\right|$.

Let $\lambda=\left(\lambda_{1}, \ldots, \lambda_{r}\right)$ be a partition of $n$. Denote

$$
W_{\lambda}^{\prime}=W_{\lambda_{1}}^{\prime} \uplus \ldots \uplus W_{\lambda_{r}}^{\prime}
$$

to be the disjoint union of subsets of $V(G)$ such that for $1 \leq i \leq r, W_{\lambda_{i}}^{\prime}$ is a subset of $V(G)$ of size $\lambda_{i}$ and $W_{\lambda_{i}}^{\prime} \cap W_{\lambda_{j}}^{\prime}=\emptyset$ for all $i \neq j$. Thus, $W_{\lambda}^{\prime}$ is a set partition of $V(G)$ of type $\lambda$.

Now, for $\lambda \vdash n$ and the graph $G$, restrict the set partition $W_{\lambda}^{\prime}$ of $V(G)$ to all of the possible distinct ordered subset compositions of $V(G)$ where each distinct ordered subset composition is a unique, ordered grouping $V(G)$ as dicated by the partition $\lambda$. Denote this new "restricted set" of $W_{\lambda}^{\prime}$ as $W_{\lambda}$.

Example 2.3. Consider the graph

and the partition $\lambda=(2,2)$. Then,

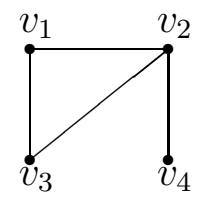

$$
\begin{aligned}
W_{\lambda}= & \\
& \left\{\left\{v_{1}, v_{2}\right\} \cup\left\{v_{3}, v_{4}\right\},\right. \\
& \left\{v_{3}, v_{4}\right\} \cup\left\{v_{1}, v_{2}\right\} \\
& \left\{v_{1}, v_{3}\right\} \cup\left\{v_{2}, v_{4}\right\} \\
& \left\{v_{2}, v_{4}\right\} \cup\left\{v_{1}, v_{3}\right\} \\
& \left\{v_{1}, v_{4}\right\} \cup\left\{v_{2}, v_{3}\right\} \\
& \left.\left\{v_{2}, v_{3}\right\} \cup\left\{v_{1}, v_{4}\right\}\right\} .
\end{aligned}
$$


Moreover, let $W_{\lambda_{i}}^{*}$ be the set of all distinct two-element subsets $\left\{v_{i}, v_{j}\right\}, i \neq j$, of $W_{\lambda_{i}}$.

Viewing each two element subset $\left\{v_{i}, v_{j}\right\} \in W_{\lambda_{i}}^{*}$ as the possible edge $v_{i} v_{j} \in E(G)$, define:

$$
P_{\lambda_{i}}= \begin{cases}\left(\left|W_{\lambda_{i}}^{*} \cap E(G)\right|+1\right) & \text { if }\left(\left|W_{\lambda_{i}}^{*} \cap E(G)\right|+1\right) \leq\left|W_{\lambda_{i}}\right| \\ \left|W_{\lambda_{i}}\right| & \text { otherwise, }\end{cases}
$$

where $P_{\lambda_{i}}=1$ if $\left(W_{\lambda_{i}}^{*} \cap E(G)\right)=\emptyset$.

For a partition $\mu=\left(\mu_{1}, \ldots, \mu_{l}\right)$, the monomial symmetric function, $m_{\mu}$, is given by: $m_{\mu}=\sum_{i_{1}<\ldots<i_{l}} x_{i_{1}}^{\mu_{1}} x_{i_{2}}^{\mu_{2}} \cdots x_{i_{l}}^{\mu_{l}}$.

Proposition 2.1. For the simple graph $G$ of order $n$,

$$
Y_{G}(x ; q, t)=\sum_{\lambda \vdash n}\left(\begin{array}{c}
n \\
\lambda_{1}, \ldots, \lambda_{r}
\end{array}\right)^{-1}\left(\sum_{W_{\lambda} \subseteq V(G)}\left(\prod_{i=1}^{r} \frac{(t ; q)_{P_{\lambda_{i}}}}{(q ; q)_{P_{\lambda_{i}}}}\right)\right) m_{\lambda}
$$

where $W_{\lambda} \subseteq V(G)$ runs over all possible distinct ordered subset compositions for the partition $\lambda=\left(\lambda_{1}, \ldots, \lambda_{r}\right) ; W_{\lambda}$ and $P_{\lambda_{i}}$ as defined above.

Proof. Since $Y_{G}(x ; q, t)$ is a symmetric function of degree $n$, it can be expressed in terms of monomial symmetric functions, $m_{\lambda}$, such that $\lambda \vdash n$. Since $k: V(G) \rightarrow \mathbb{N}$ ranges over all possible colorings of $G$, we obtain the functions $m_{\lambda}$ such that $\lambda=\left(\lambda_{1}, \ldots, \lambda_{r}\right)$ runs through all partitions of $n$, where $\lambda_{i} \equiv V_{j}, j$ ranging throughout $R(k)$ such that $\left|V_{j}\right|=\lambda_{i}$.

For $\lambda=\left(\lambda_{1}, \ldots, \lambda_{r}\right) \vdash n$, there are $\left(\begin{array}{c}n \\ \lambda_{1}, \ldots, \lambda_{r}\end{array}\right)$ possible distinct general colorings within the basic coloring $k_{\lambda}$; sending $\lambda_{i}$-many vertices to the same color $j \in R(k), 1 \leq i \leq r$, and where the vertices of $\lambda_{i}$ are sent to a distinct color from those of $\lambda_{m}, \forall i \neq m$.

Since $W_{\lambda}=W_{\lambda_{1}} \uplus \ldots \uplus W_{\lambda_{r}}$ partitions the vertices of $V(G)$ into all possible disjoint subsets such that $\left|W_{\lambda_{i}}\right|=\lambda_{i}$, and since $W_{\lambda} \subseteq V(G)$ runs over all distinct ordered $W_{\lambda}$ (with respect to the composition of $W_{\lambda_{i}}, \forall i$ ), we obtain all distinct general colorings $k_{\lambda}^{i}$ of $G$ within $k_{\lambda}$. Since the "specific" colorings within each $k_{\lambda}^{m}$ have the same coefficient (ref. Example 2.2), we may consider the coefficient of $m_{\lambda}, \lambda \vdash n$, via the general coefficients for a coloring of type $\lambda, k_{\lambda}$, with respect to the individual coefficients for each $k_{\lambda}^{m}$. Since $l(\lambda)=|R(k)|$ for the coloring $k$, one can see by comparing the $m_{i}$ to the $P_{\lambda_{i}}$ that these terms coincide. Thus, the coefficent of the monomial $m_{\lambda}$ in $Y_{G}(x ; q, t)$ is equal to

$$
\left(\begin{array}{c}
n \\
\lambda_{1}, \ldots, \lambda_{r}
\end{array}\right)^{-1}\left(\sum_{W_{\lambda} \subseteq V(G)}\left(\prod_{i=1}^{r} \frac{(t ; q)_{P_{\lambda_{i}}}}{(q ; q)_{P_{\lambda_{i}}}}\right)\right) .
$$


Example 2.4. For the graph $G$ of Example 2.2, it is easily seen that

$$
\begin{aligned}
& Y_{G}(x ; q, t)=\frac{(t ; q)^{4}}{(q ; q)^{4}} m_{(1,1,1,1)}+\frac{(t ; q)_{4}}{(q ; q)_{4}} m_{(4)} \\
& +\left(\frac{3}{4} \frac{(t ; q)_{3}(t ; q)}{(q ; q)_{3}(q ; q)}+\frac{1}{4} \frac{(t ; q)_{2}(t ; q)}{(q ; q)_{2}(q ; q)}\right) m_{(3,1)} \\
& +\left(\frac{2}{3} \frac{(t ; q)_{2}(t ; q)(t ; q)}{(q ; q)_{2}(q ; q)(q ; q)}+\frac{1}{3} \frac{(t ; q)^{3}}{(q ; q)^{3}}\right) m_{(2,1,1)} \\
& +\left(\frac{2}{3} \frac{(t ; q)_{2}(t ; q)}{(q ; q)_{2}(q ; q)}+\frac{1}{3} \frac{(t ; q)_{2}(t ; q)_{2}}{(q ; q)_{2}(q ; q)_{2}}\right) m_{(2,2)} .
\end{aligned}
$$

\section{Some Properties of $Y_{G}(x ; q, t)$.}

In this section, we will explore some of the basic properties and correlations between a finite, simple graph $G$ and the symmetric function $Y_{G}(x ; q, t)$.

Proposition 3.1. Let $G$ be a simple graph. $G$ has order $d$ and size $s$ if the multiplicity of the term

$$
\frac{(t ; q)_{2}(t ; q)^{(d-2)}}{(q ; q)_{2}(q ; q)^{(d-2)}} m_{(2,1(d-2)}
$$

in $Y_{G}(x ; q, t)$ is $\frac{2 s}{d(d-1)}$.

Proof. Let $G$ be a graph of order $d$ and size $s$. The multiplicity of the term (6) in $Y_{G}(x ; q, t)$ corresponds to $\left(\begin{array}{c}d \\ 2,1^{(d-2)}\end{array}\right)^{-1}$ multiplied by the number of pairs of vertices $\left\{v_{i}, v_{j}\right\} \in V(G)$ such that $v_{i} v_{j} \in E(G)$ (where $P_{(2)}=2$ ) multiplied by $(d-2)$ ! :

For $\left\{v_{i}, v_{j}\right\} \in V(G)$ such that $v_{i} v_{j} \in E(G)$, consider the number of possible general distinct colorings $k_{\left(2,1^{(d-2)}\right)}^{m}: V(G) \rightarrow \mathbb{N}$ of type $\left(2,1^{(d-2)}\right)$ such that $k_{(2,1(d-2))}^{m}\left(v_{i}\right)=$ $k_{\left(2,1^{(d-2)}\right)}^{m}\left(v_{j}\right)$ and such that $k_{\left(2,1^{(d-2)}\right)}^{m}$ distinguishes all remaining vertices in $V(G)$ from each other and from $v_{i}$ and $v_{j}$. Since $s(G)=s$, there are $s$ possible such two element subsets $\left\{v_{i}, v_{j}\right\}$ of $V(G)$. For each of these subsets, since $o(G)=d$, there are $(d-2)$ remaining vertices in $V(G) \backslash\left\{v_{i}, v_{j}\right\}$. Thus, there are $(d-2)$ ! different general distinct colorings $k_{\left(2,1^{(d-2)}\right)}^{m}$ distinguishing among $V(G) \backslash\left\{v_{i}, v_{j}\right\}$ and $\left\{v_{i}, v_{j}\right\}$. Hence, the multiplicity of the desired term is

$$
\left(\begin{array}{c}
d \\
2,1^{(d-2)}
\end{array}\right)^{-1} s(d-2) !=\frac{2 s}{d(d-1)} \text {. }
$$

Remark 3.1. Conversely to Proposition 3.1, consider $Y_{G}(x ; q, t)$ in which the term

$$
\frac{(t ; q)_{2}(t ; q)^{r}}{(q ; q)_{2}(q ; q)^{(r}} m_{\left(2,1^{r}\right)}
$$

appears. 
Note that the monomial symmetric function $m_{\left(2,1^{r}\right)}$, for some $r \geq 0$, appears in $Y_{G}(x ; q, t)$ if and only if $o(G)=2+r$ since $\left(2,1^{r}\right) \vdash(2+r)$. Furthermore, by Proposition 2.1, the coefficient of the monomial $m_{\left(2,1^{r}\right)}$ is equal to

$$
\left(\begin{array}{c}
2+r \\
2,1^{r}
\end{array}\right)^{-1}\left(\sum_{W_{\lambda} \subseteq V(G)}\left(\prod_{i=1}^{2+r} \frac{(t ; q)_{P_{\lambda_{i}}}}{(q ; q)_{P_{\lambda_{i}}}}\right)\right) .
$$

For

$$
\sum_{W_{\lambda} \subseteq V(G)}\left(\prod_{i=1}^{2+r} \frac{(t ; q)_{P_{\lambda_{i}}}}{(q ; q)_{P_{\lambda_{i}}}}\right),
$$

we have $P_{\lambda_{1}}=2$ and $P_{\lambda_{2}}=\ldots=P_{\lambda_{r}}=1$. Hence, by definition of $P_{\lambda_{i}}$, and since $\left|W_{\lambda_{i}}\right|=2$, it follows that the multiplicity of (6) is:

$$
\begin{aligned}
& \left(\begin{array}{c}
2+r \\
2,1^{r}
\end{array}\right)^{-1} \cdot|E(G)| \cdot r ! \\
& =\frac{2}{(2+r) !} \cdot|E(G)| \cdot r ! \\
& =\frac{2|E(G)|}{(2+r)(1+r)} .
\end{aligned}
$$

Therefore, given the multiplicity of (6), we may recover $|E(G)|$.

Proposition 3.2. Let $G$ and $H$ be graphs with degree sequences $\operatorname{deg}(G)$ and $\operatorname{deg}(H)$, respectively. Then $o(G)=o(H)=d, s(G)=s(H) \leq d$, and $\operatorname{deg}(G)=\operatorname{deg}(H)$ if and only if the multiplicity of the term

$$
\frac{(t ; q)_{2}(t ; q)^{(d-2)}}{(q ; q)_{2}(q ; q)^{(d-2)}} m_{(2,1(d-2))}
$$

is $\leq \frac{2}{(d-1)}$ and is equal in both $Y_{G}(x ; q, t)$ and $Y_{H}(x ; q, t)$ and if the coefficients of $m_{((d-1), 1)}$ in $Y_{G}(x ; q, t)$ and $Y_{H}(x ; q, t)$ are equal.

Proof. $(\Rightarrow)$ Suppose that $o(G)=o(H)=d, s(G)=s(H) \leq d$, and $\operatorname{deg}(G)=$ $\operatorname{deg}(H)$. Let $\operatorname{deg}(G)=\left(\beta_{1}, \ldots, \beta_{n}\right)=\operatorname{deg}(H) ; \beta_{1} \geq \ldots \geq \beta_{n}, n \leq d$, and $\sum_{i=1}^{n} \beta_{i}=$ $2 s(G)$. By Proposition 3.1, we know that the multiplicity of the term (6) is equal in both $Y_{G}$ and $Y_{H}$ and is $\leq \frac{2}{(d-1)}$. By the definitions of $Y_{G}(x ; q, t)$ and $Y_{H}(x ; q, t)$, the coefficients of $m_{((d-1), 1)}$ are given by

$$
\begin{aligned}
& \left(\begin{array}{c}
d \\
(d-1), 1
\end{array}\right)^{-1}\left(\sum_{W_{\lambda} \subseteq V(G)}\left(\prod_{i=1}^{2} \frac{(t ; q)_{P_{\lambda_{i}}}}{(q ; q)_{P_{\lambda_{i}}}}\right)\right) \\
& =\frac{1}{d}\left(\sum_{W_{((d-1), 1)} \subset V(G)} \frac{(t ; q)_{P_{(d-1)}}(t ; q)_{P_{(1)}}}{(q ; q)_{P_{(d-1)}}(q ; q)_{P_{(1)}}}\right) .
\end{aligned}
$$


Note that there are $d$-many distinct subsets $W_{((d-1), 1)}=W_{(d-1)} \uplus W_{(1)}$ of $V(G)$ (resp., $V(H)$ ). Moreover, note that each $\beta_{i} \in \operatorname{deg}(G)$ (resp., $\operatorname{deg}(H)$ ) directly corresponds to one vertex $v_{j} \in V(G)$, where $\beta_{i}$ indicates the degree of the vertex $v_{j}, d\left(v_{j}\right)=\beta_{i}$. Thus, sending the vertex $v_{j}$ to $W_{(1)}$ amounts to removing all edges from $E(G)$ (resp., $E(H)$ ) which are incident with the vertex $v_{j}$ in the computation of $\left|W_{(d-1)}^{*} \cap E(G)\right|+1=P_{(d-1)}, W_{(d-1)}=$ $V(G) \backslash\left\{v_{j}\right\}$. This implies that $\left|W_{(d-1)}^{*} \cap E(G)\right|=|E(G)|-\beta_{i}$ (resp. for $H$ ). Repeating this for each $\beta_{i} \in \operatorname{deg}(G)=\operatorname{deg}(H)$ and the corresponding two vertices (one for $\operatorname{deg}(G)$ and possibly a different one for $\operatorname{deg}(H))$ gives the coefficients of $m_{((d-1), 1)}$ in $Y_{G}(x ; q, t)$ and $Y_{H}(x ; q, t)$ to be equal.

$(\Leftarrow)$ From Proposition 3.1, the multiplicity of the term $(6)$ being equal and $\leq \frac{2}{(d-1)}$ in $Y_{G}$ and $Y_{H}$ tells us that $o(G)=o(H)=d$ and that $s(G)=s(H) \leq d$.

Suppose that the coefficients of the term $m_{((d-1), 1)}$ in both $Y_{G}(x ; q, t)$ and $Y_{H}(x ; q, t)$ are equal. We must show that $\operatorname{deg}(G)=\operatorname{deg}(H)$. For $1 \leq l \leq(d-1)$, consider the multiplicity $K_{l}$ of the term

$$
\frac{(t ; q)_{l}(t ; q)}{(q ; q)_{l}(q ; q)} m_{((d-1), 1)}
$$

in $Y_{G}(x ; q, t)$ and $Y_{H}(x ; q, t)$.

Suppose that $l=(d-1)$. Then there exists $K_{(d-1)}$ vertices in $V(G)$ such that $\mid W_{(d-1)}^{*} \cap$ $E(G) \mid=(d-1)$ or $(d-2)$, and similarly for $V(H)$. We need to show that the number of vertices in $V(H)$ such that $\left|W_{(d-1)}^{*} \cap E(G)\right|=(d-1)$ (resp. $(d-2)$ ) is equal to the number of vertices in $V(H)$ such that $\left|W_{(d-1)}^{*} \cap E(H)\right|=(d-1)$ (resp. $\left.(d-2)\right)$.

Note that the multiplicity of

$$
\frac{(t ; q)_{(d-1)}(t ; q)}{(q ; q)_{(d-1)}(q ; q)} m_{((d-1), 1)}
$$

corresponds to the number of vertices in $V(G)$ and $V(H)$ such that $d\left(v_{i}\right)=1$ or $d\left(v_{i}\right)=0$. Consider the vertices $v_{i} \in V(G)$ and $v_{j} \in V(H)$, for which $W_{(1)}=\left\{v_{i}\right\}$ and $W_{(1)}=\left\{v_{j}\right\}$ in $W_{((d-1), 1)}$, such that $P_{(d-1)} \leq(d-2)$. For each $W_{((d-1), 1)} \subset V(G)$ and $W_{((d-1), 1)} \subset V(H)$ such that $P_{(d-1)}$ is equal for both $V(G)$ and $V(H)$ and $P_{(d-1)} \leq(d-2)$, we have that $\left|W_{(d-1)}^{*} \cap E(G)\right|=\left(P_{(d-1)}-1\right)=\left|W_{(d-1)}^{*} \cap E(H)\right|$, by definition of $P_{(d-1)}$. Since the multiplicity of the coefficient of

$$
\frac{(t ; q)_{P_{(d-1)}}(t ; q)}{(q ; q)_{P_{(d-1)}}(q ; q)} m_{((d-1), 1)}
$$

in $Y_{G}$ and $Y_{H}$ is equal, the number of vertices $v_{i} \in V(G)$ and $v_{j} \in V(H)$ such that $d\left(v_{i}\right)=d\left(v_{j}\right)=s-P_{(d-1)}$ must be equal. (Note: $P_{(d-1)}+1=s-d\left(v_{i}\right)+1$.) Thus, since $o(G)=o(H), s(G)=s(H)$, and $\sum \operatorname{deg}(G)=\sum \operatorname{deg}(H)$, the number of vertices with degree 0 in $G$ equals the number of vertices with degree 0 in $H$ and, similarly, the number of vertices with degree 1 in $G$ equals the number of vertices with degree 1 in $H$. Therefore, $\operatorname{deg}(G)=\operatorname{deg}(H)$. 
Proposition 3.3. Let $G$ be a simple graph of order $d$. Any induced subgraph of $G, G_{I}$, of order $(d-1)$ is connected if and only if the multiplicity of the term

$$
\frac{(t ; q)_{(d-1)}(t ; q)}{(q ; q)_{(d-1)}(q ; q)} m_{((d-1), 1)}
$$

in $Y_{G}(x ; q, t)$ is one.

Proof. $(\Rightarrow)$ Suppose that any induced subgraph of $G, G_{I}$, of order $(d-1)$ is connected. Then, $\left|E\left(G_{I}\right)\right| \geq(d-2)$. Hence, for all possible subsets $W_{(d-1)} \subset V(G), W_{(d-1)} \subset$ $W_{((d-1), 1)}$, it follows that $P_{(d-1)}=(d-1)$. Hence, the multiplicity term $(7)$ in $Y_{G}(x ; q, t)$ is one.

$(\Leftarrow)$ Suppose that the multiplicity of term $(7)$ in $Y_{G}(x ; q, t)$ is one. Then, for all possible $(d-1)$-element subsets $W_{(d-1)} \subset V(G),\left|W_{(d-1)}^{*} \cap E(G)\right| \geq(d-2)$. Therefore, every induced subgraph $G_{I}$ of order $(d-1)$ must be connected.

Remark 3.2. By Proposition 3.3, for a graph $G$ of order $d$, if the multiplicity of (7) is one in $Y_{G}(x ; q, t)$, then $G$ is not a tree.

Proposition 3.4. Let $G$ be a simple graph. $G$ has order $d$ and is a cycle of size $d$ if and only if the multiplicity of the term

$$
\frac{(t ; q)_{(d-1)}(t ; q)}{(q ; q)_{(d-1)}(q ; q)} m_{((d-1), 1)}
$$

in $Y_{G}(x ; q, t)$ is one and the multiplicity of the term

$$
\frac{(t ; q)_{2}(t ; q)^{(d-2)}}{(q ; q)_{2}(q ; q)^{(d-2)}} m_{\left(2,1^{(d-2)}\right)}
$$

is $\frac{2}{(d-1)}$.

Proof. $(\Rightarrow)$ If $o(G)=s(G)=d$, we know from Proposition 3.1 that the multiplicity of the term (6) is $\frac{2}{(d-1)}$. Consider the multiplicity of the term (7). Since $G$ is a cycle of length $d$ and $o(G)=s(G)=d$, we know that $d\left(v_{i}\right)=2$ for all $v_{i} \in V(G)$. Thus, the number of subsets $W_{\lambda} \subseteq V(G), W_{\lambda}=W_{(d-1)} \uplus W_{(1)}$, such that $P_{(d-1)}=(d-1)$ and $P_{(1)}=1$ is exactly $d$ many, since any choice of $(d-1)$ vertices is connected by $(d-2)$ edges. This implies that the multiplicity of the desired term is

$$
d\left(\begin{array}{c}
d \\
(d-1), 1
\end{array}\right)^{-1}=1 .
$$

$(\Leftarrow)$ From Proposition 3.1 and Remark 3.1, if the multiplicity of the term $(6)$ is $\frac{2}{(d-1)}$ for some $d$, we know that $G$ has order and size $d$. By Proposition 3.3, the multiplicity of term $(7)$ being one implies that any $(d-1)$ element subset of $V(G)$ is connected. Since $o(G)=s(G)=d$, the only connected graph fitting this description is a cycle of length $d$. 


\section{4. $\mathrm{Y}_{\mathrm{G}}(\mathrm{x} ; \mathrm{q}, \mathrm{t})$ and Macdonald Polynomials.}

Denote the ring of symmetric functions over the field $\mathbb{F}$ as $\Lambda_{\mathbb{F}}$ and let $\Lambda_{\mathbb{F}}^{n}$ denote its $n^{\text {th }}$ graded space. The space $\Lambda_{\mathbb{F}}^{n}$ consists of all symmetric functions of total degree $n \in \mathbb{Z}$, indexed by the partitions $\lambda=\left(\lambda_{1}, \ldots, \lambda_{r}\right)$ for which $\sum_{i} \lambda_{i}=n$. Five important bases of $\Lambda_{\mathbb{F}}^{n}$ are: the monomial symmetric functions $m_{\lambda}$, the elementary symmetric functions $e_{\lambda}=e_{\lambda_{1}} \cdots e_{\lambda_{r}}$, the complete symmetric functions $h_{\lambda}$, the Schur functions $s_{\lambda}$, and the power sum symmetric functions $p_{\lambda}=p_{\lambda_{1}} \cdots p_{\lambda_{r}}$. Of these five bases, all except the power sum symmetric functions are $\mathbb{Z}$-bases; the power sum symmetric functions are a $\mathbb{Q}$-basis.

Let $H=\mathbb{Q}(q, t)$ be the field of rational functions in $q$ and $t$. In 1988, Macdonald introduced a new class of two-parameter symmetric functions $P_{\lambda}(q, t)$, over the ring $\Lambda_{H}$, which generalize several classes of symmetric functions. In particular, taking $q=t$ we obtain the Schur functions, setting $t=1$ we have the monomial symmetric functions, and letting $q=0$ gives the Hall-Littlewood functions.

We know from [4] that the $\left(P_{\lambda}\right)$ are a basis of $\Lambda_{H}^{n}$. Further, with respect to the scalar product:

$$
<p_{\lambda}, p_{\mu}>=\delta_{\lambda, \mu} \prod_{i} i^{m_{i}} m_{i} ! \prod_{j=1}^{l(\lambda)} \frac{1-q^{\lambda_{j}}}{1-t^{\lambda_{j}}}
$$

we have that

$$
<P_{\lambda}, P_{\mu}>=0 \quad \text { if } \quad \lambda \neq \mu,
$$

where $m_{i}$ denotes the multiplicity of $i$ in $\lambda$ and $l(\lambda)$ denotes the length of $\lambda$. We also know that for each $\lambda$, there exists a unique $P_{\lambda}(q, t)$ such that:

$$
P_{\lambda}=m_{\lambda}+\sum_{\mu<\lambda} c_{\lambda \mu} m_{\mu} \quad \text { where } \quad c_{\lambda \mu} \in \mathbb{Q}(q, t) .
$$

Define:

$$
Q_{\lambda}=\frac{P_{\lambda}}{<P_{\lambda}, P_{\lambda}>} .
$$

Then, the bases $\left(P_{\lambda}\right)$ and $\left(Q_{\lambda}\right)$ of $\Lambda_{H}^{n}$ are dual to each other, $\left\langle Q_{\lambda}, P_{\mu}\right\rangle=\delta_{\lambda, \mu}$, and from [4], for $\gamma=(n)$ :

$$
Q_{(n)}=\sum_{|\lambda|=n} \prod_{i} \frac{1}{i^{m_{i}} m_{i} !} \prod_{j=1}^{l(\lambda)} \frac{1-t^{\lambda_{j}}}{1-q^{\lambda_{j}}} p_{\lambda}
$$

where we set $Q_{0}=1$ and $Q_{-m}=0$ for $m \in \mathbb{Z}^{+}$.

There turns out to be an interesting connection between our two parameter chromatic symmetric function $Y_{G}(x ; q, t)$ and the Macdonald polynomials $Q_{\lambda}$. We motivate this connection via the following definitions and proposition.

The complete graph of order $n$, denoted $K^{n}$, is the graph $G$ which has size $\left(\begin{array}{l}n \\ 2\end{array}\right)$; every two vertices in $V(G)$ are adjacent. We know from [4] that for $n \in \mathbb{Z}^{+}$, the Macdonald polynomial

$$
Q_{(n)}=\sum_{\lambda \vdash n} \frac{(t ; q)_{\lambda}}{(q ; q)_{\lambda}} m_{\lambda}
$$


where we define

$$
\frac{(t ; q)_{\lambda}}{(q ; q)_{\lambda}}=\prod_{i=1}^{r} \frac{(t ; q)_{\lambda_{i}}}{(q ; q)_{\lambda_{i}}}
$$

for $\lambda=\left(\lambda_{1}, \ldots, \lambda_{r}\right) \vdash n$.

The following proposition is immediate.

Proposition 4.1. Let $G$ be the complete graph of order $n, G=K^{n}$, for $n \in \mathbb{Z}^{+}$. Then

$$
Y_{G}(x ; q, t)=Q_{(n)}(x ; q, t)
$$

From [3], we have the following combinatoral formula for a two-row Macdonald polynomial $Q_{\lambda}, \lambda=\left(\lambda_{1}, \lambda_{2}\right)$ :

$$
Q_{\left(\lambda_{1}, \lambda_{2}\right)}=\sum_{i=0}^{\lambda_{2}} a_{i}^{\lambda_{1}-\lambda_{2}} Q_{\left(\lambda_{1}+i\right)} Q_{\left(\lambda_{2}-i\right)}
$$

where

$$
a_{i}^{\lambda_{1}-\lambda_{2}}=\left(\frac{\left(t^{-1} ; q\right)_{i}\left(q^{\lambda_{1}-\lambda_{2}} ; q\right)_{i}\left(1-q^{\lambda_{1}-\lambda_{2}+2 i}\right)}{(q ; q)_{i}\left(q^{\lambda_{1}-\lambda_{2}+1} t ; q\right)_{i}\left(1-q^{\lambda_{1}-\lambda_{2}}\right)}\right) t^{i}
$$

and $a_{0}^{\lambda_{1}-\lambda_{2}}=1$

Using the symmetric function $Y_{G}(x ; q, t)$, we give a graphical analogue of this two-row formula for any partition $\lambda=\left(\lambda_{1}, \lambda_{2}\right)$.

Let $G$ be the complete graph of order $\left(\lambda_{1}+\lambda_{2}\right), G=K^{\left(\lambda_{1}+\lambda_{2}\right)}$. Then, $V(G)=$ $\left\{v_{1}, \ldots, v_{\lambda_{1}+\lambda_{2}}\right\}$. Denote $W_{i}$ to be the subset of $V(G)$ containing vertices $\left\{v_{j}\right\}$ for $1 \leq$ $j \leq i$ :

$$
W_{i}=\left\{v_{1}, \ldots, v_{i}\right\} .
$$

Denote $W_{i}^{c}$ to be the subset of $V(G)$ containing the vertices $\left\{v_{m}\right\}$ such that $(i+1) \leq$ $m \leq\left(\lambda_{1}+\lambda_{2}\right)$

$$
W_{i}^{c}=\left\{v_{(i+1)}, \ldots, v_{\left(\lambda_{1}+\lambda_{2}\right)}\right\}
$$

and set $W_{0}=\emptyset$.

Let $G\left[V \backslash W_{i}\right]$ denote the subgraph of $G=K^{\left(\lambda_{1}+\lambda_{2}\right)}$ obtained by deleting the vertices in $W_{i} \subseteq V(G)$ and all edges in $E(G)$ which are incident with them.

Theorem 4.1. Let $G=K^{\left(\lambda_{1}+\lambda_{2}\right)}$. For the partition $\lambda=\left(\lambda_{1}, \lambda_{2}\right)$,

$$
Q_{\lambda}=Q_{\left(\lambda_{1}, \lambda_{2}\right)}=\sum_{i=0}^{\lambda_{2}} a_{\left(\lambda_{2}-i\right)}^{\lambda_{1}-\lambda_{2}} Y_{G\left[V \backslash W_{i}\right]}(x ; q, t) Y_{G\left[V \backslash W_{i}^{c}\right]}(x ; q, t)
$$

where

$$
Y_{G\left[V \backslash W_{i}^{c}\right]}(x ; q, t)=1 \quad \text { if } \quad V \backslash W_{i}^{c}=\emptyset
$$

and where $a_{\left(\lambda_{2}-i\right)}^{\lambda_{1}-\lambda_{2}}$ is defined above. 
Proof. Note that the complete graph $G=K^{\left(\lambda_{1}+\lambda_{2}\right)}$ contains all of the complete graphs $K^{l}$ for $0<l<\left(\lambda_{1}+\lambda_{2}\right)$. Since $G\left[V \backslash W_{i}\right]$ is the complete graph on $\left(\lambda_{1}+\lambda_{2}-i\right)$-many vertices, $G\left[V \backslash W_{i}\right]=K^{\left(\lambda_{1}+\lambda_{2}-i\right)}$, it follows that $Y_{G\left[V \backslash W_{i}\right]}(x ; q, t)=Q_{\left(\lambda_{1}+\lambda_{2}-i\right)}$. Similarly, $G\left[V \backslash W_{i}^{c}\right]=K^{i}$ which in turn implies that $Y_{G\left[V \backslash W_{i}^{c}\right]}(x ; q, t)=Q_{(i)}$. Expressing (8) as

$$
Q_{\left(\lambda_{1}+\lambda_{2}\right)}=\sum_{i=0}^{\lambda_{2}} a_{\left(\lambda_{2}-i\right)}^{\lambda_{1}-\lambda_{2}} Q_{\left(\lambda_{1}+\lambda_{2}-i\right)} Q_{(i)}
$$

the result follows.

Example 4.1. Consider the expression of the two-row Macdonald polynomial $Q_{(3,2)}$. By Theorem 4.1, we have

$$
Q_{(3,2)}=\sum_{i=0}^{2} a_{(2-i)} Y_{G\left[V \backslash W_{i}\right]}(x ; q, t) Y_{G\left[V \backslash W_{i}^{c}\right]}(x ; q, t)
$$

where $G=K^{5}$. Thus,

$$
\begin{aligned}
Q_{(3,2)}= & a_{(2)} Y_{G\left[V \backslash W_{0}\right]}(x ; q, t) Y_{G\left[V \backslash W_{0}^{c}\right]}(x ; q, t) \\
& +a_{(1)} Y_{G\left[V \backslash W_{1}\right]}(x ; q, t) Y_{G\left[V \backslash W_{1}^{c}\right]}(x ; q, t) \\
& +a_{(0)} Y_{G\left[V \backslash W_{2}\right]}(x ; q, t) Y_{G\left[V \backslash W_{2}^{c}\right]}(x ; q, t) .
\end{aligned}
$$
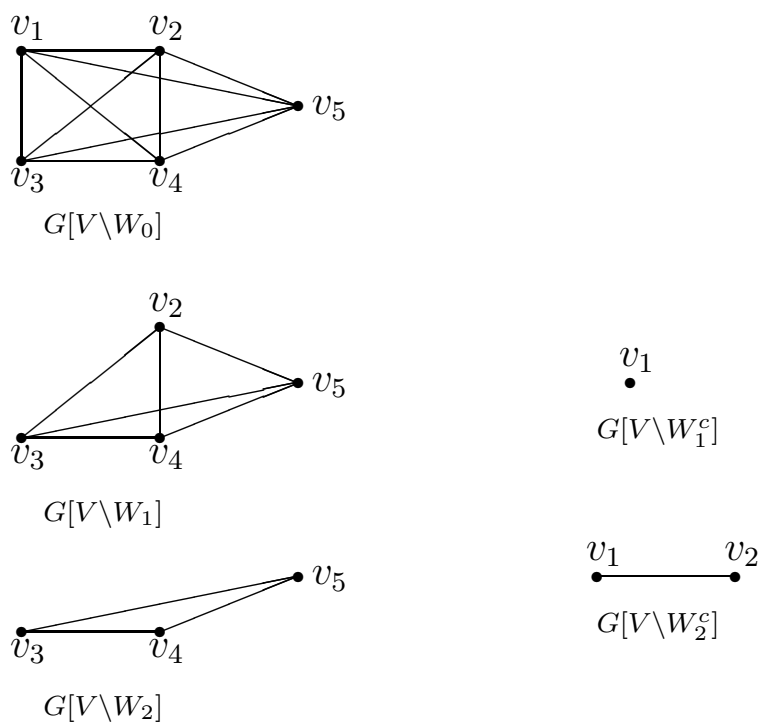

Computing the respective $Y_{G\left[V \backslash W_{i}\right]}(x ; q, t)$ and $Y_{G\left[V \backslash W_{i}^{c}\right]}(x ; q, t)$ for $0 \leq i \leq 2$ yields:

$$
Q_{(3,2)}=\left(\frac{\left(t^{-1} ; q\right)_{2}}{\left(q^{2} t ; q\right)_{2}} \frac{\left(1-q^{5}\right)}{(1-q)} t^{2}\right) Q_{(5)}+\left(\frac{\left(t^{-1} ; q\right)}{\left(q^{2} t ; q\right)} \frac{\left(1-q^{3}\right)}{(1-q)} t\right) Q_{(4)} Q_{(1)}+Q_{(3)} Q_{(2)} \text {. }
$$

For the parameter $\alpha$, the Jack symmetric functions, $J_{\lambda}$, are defined by:

$$
J_{\lambda}=Q_{\lambda}(\alpha)=\lim _{t \rightarrow 1} Q_{\lambda}\left(t^{\alpha}, t\right)
$$


where we set $q=t^{\alpha}$ in $Q_{\lambda}(x ; q, t)$.

In [3], we have a formula for the Jack functions $J_{\lambda}, \lambda=\left(\lambda_{1}, \lambda_{2}\right)$ :

$$
J_{\left(\lambda_{1}, \lambda_{2}\right)}=Q_{\left(\lambda_{1}, \lambda_{2}\right)}(\alpha)=\sum_{i=0}^{\lambda_{2}} a_{i}^{\lambda_{1}-\lambda_{2}}(\alpha) Q_{\left(\lambda_{1}+i\right)}(\alpha) Q_{\left(\lambda_{2}-i\right)}(\alpha)
$$

where

$$
\begin{aligned}
a_{i}^{\lambda_{1}-\lambda_{2}}= & (-1)^{i}\left(\frac{(1-\alpha) \cdots(1-(i-1) \alpha)}{i !}\right) \\
& \cdot\left(\frac{\left(\lambda_{1}-\lambda_{2}+1\right) \cdots\left(\lambda_{1}-\lambda_{2}+i-1\right)\left(\lambda_{1}-\lambda_{2}+2 i\right)}{\left(1+\left(\lambda_{1}-\lambda_{2}+1\right) \alpha\right) \cdots\left(1+\left(\lambda_{1}-\lambda_{2}+i\right) \alpha\right)}\right) .
\end{aligned}
$$

Set $q=t^{\alpha}$ in the two-parameter symmetric function $Y_{G}(x ; q, t)$. Define

$$
Y_{G}(\alpha)=\lim _{t \rightarrow 1} Y_{G}\left(x ; t^{\alpha}, t\right) .
$$

Similar to Theorem 4.1, we obtain a graphical analogue for the expansion of the tworow Jack symmetric functions $J_{\left(\lambda_{1}, \lambda_{2}\right)}$ using $Y_{G}(\alpha)$.

Corollary 4.1. Let $G=K^{\left(\lambda_{1}+\lambda_{2}\right)}$. For the partition $\lambda=\left(\lambda_{1}, \lambda_{2}\right)$,

$$
J_{\left(\lambda_{1}, \lambda_{2}\right)}=Q_{\left(\lambda_{1}, \lambda_{2}\right)}(\alpha)=\sum_{i=0}^{\lambda_{2}} a_{\left(\lambda_{2}-i\right)}^{\lambda_{1}-\lambda_{2}}(\alpha) Y_{G\left[V \backslash W_{i}\right]}(\alpha) Y_{G\left[V \backslash W_{i}^{c}\right]}(\alpha)
$$

where

$$
Y_{G\left[V \backslash W_{i}^{c}\right]}(\alpha)=1 \quad \text { if } \quad V \backslash W_{i}^{c}=\emptyset,
$$

$a_{\left(\lambda_{2}-i\right)}^{\lambda_{1}-\lambda_{2}}$ defined above, and $a_{0}^{\lambda_{1}-\lambda_{2}}(\alpha)=1$.

\section{The Symmetric Function $\mathrm{Y}_{\mathrm{G}}^{\mathrm{c}}(\mathrm{x} ; \mathrm{q}, \mathrm{t})$.}

We now introduce the "complement," $Y_{G}^{c}(x ; q, t)$, of the two-parameter symmetric function $Y_{G}(x ; q, t)$.

Define

$$
m_{i}^{c}= \begin{cases}\left(\left(\begin{array}{c}
V_{i} \\
2
\end{array}\right)-m_{i}(k)+1\right) & \text { if }\left(\left(\begin{array}{c}
V_{i} \\
2
\end{array}\right)-m_{i}(k)+1\right) \leq V_{i} \\
V_{i} & \text { otherwise. }\end{cases}
$$

\section{Definition 5.1.}

$$
Y_{G}^{c}(x ; q, t)=\sum_{k}\left(\begin{array}{c}
n \\
V_{1}, V_{2}, \ldots
\end{array}\right)^{-1}\left(\prod_{i \in R(k)} \frac{(t ; q)_{m_{i}^{c}}}{(q ; q)_{m_{i}^{c}}}\right) x^{k}
$$

where $k$ ranges over all colorings of $G$. 
Now, define

$$
P_{\lambda_{i}}^{c}= \begin{cases}\left(\left|W_{\lambda_{i}}^{*}\right|-\left|W_{\lambda_{i}}^{*} \cap E(G)\right|+1\right) & \text { if }\left(\left|W_{\lambda_{i}}^{*}\right|-\left|W_{\lambda_{i}}^{*} \cap E(G)\right|+1\right) \leq\left|W_{\lambda_{i}}\right| \\ \left|W_{\lambda_{i}}\right| & \text { otherwise. }\end{cases}
$$

Note that $\left|W_{\lambda_{i}}^{*}\right|=\left(\begin{array}{c}\left|W_{\lambda_{i}}\right| \\ 2\end{array}\right)$.

Proposition 5.1. For the simple graph $G$ of order $n$,

$$
Y_{G}^{c}(x ; q, t)=\sum_{\lambda \vdash n}\left(\begin{array}{c}
n \\
\lambda_{1}, \ldots, \lambda_{r}
\end{array}\right)^{-1}\left(\sum_{W_{\lambda} \subseteq V(G)}\left(\prod_{i=1}^{r} \frac{(t ; q)_{P_{\lambda_{i}}^{c}}}{(q ; q)_{P_{\lambda_{i}}^{c}}}\right)\right) m_{\lambda}
$$

where $W_{\lambda} \subseteq V(G)$ runs over all possible distinct ordered subset compositions for the partition $\lambda=\left(\lambda_{1}, \ldots, \lambda_{r}\right) ; W_{\lambda}$ and $P_{\lambda_{i}}^{c}$ defined above.

Proof. Similar to the proof of Proposition 2.1, comparing the $m_{i}^{c}$ to the $P_{\lambda_{i}}^{c}$ for the basic coloring $k_{\lambda}$ of type $\lambda=\left(\lambda_{1}, \ldots, \lambda_{r}\right)$, and noting that $\left|W_{\lambda_{i}}^{*}\right|=\left(\begin{array}{c}\left|W_{\lambda_{i}}\right| \\ 2\end{array}\right)$, we see that the coefficient of $m_{\lambda}$ in $Y_{G}^{c}(x ; q, t)$ is equal to

$$
\left(\begin{array}{c}
n \\
\lambda_{1}, \ldots, \lambda_{r}
\end{array}\right)^{-1}\left(\sum_{W_{\lambda} \subseteq V(G)}\left(\prod_{i=1}^{r} \frac{(t ; q)_{P_{\lambda_{i}}^{c}}}{(q ; q)_{P_{\lambda_{i}}^{c}}}\right)\right) .
$$

Let $G$ be a simple, finite graph of order $n$. Then, the complement of the graph $G$, denoted $G^{c}$, is the graph of order $n$ such that $v_{i} v_{j} \in E\left(G^{c}\right)$ if and only if $v_{i} v_{j} \notin E(G)$. Thus, if $G$ has size $d$, it follows that $G^{c}$ has size $\left(\left(\begin{array}{l}n \\ 2\end{array}\right)-d\right)$.

Theorem 5.1. $Y_{G}^{c}(x ; q, t)=Y_{G^{c}}(x ; q, t)$.

Proof. Let $G$ be a simple graph of order $n$ with complement $G^{c}$. We want to show that for $\lambda \vdash n$, the coefficient of the monomial symmetric function $m_{\lambda}$ in $Y_{G}^{c}(x ; q, t)$ and $Y_{G^{c}}(x ; q, t)$ are equal.

For $\lambda \vdash n$, the coefficient of $m_{\lambda}$ in $Y_{G}^{c}(x ; q, t)$ is given by (13) and the coefficient of $m_{\lambda}$ is $Y_{G^{c}}(x ; q, t)$ is given by

$$
\left(\begin{array}{c}
n \\
\lambda_{1}, \ldots, \lambda_{r}
\end{array}\right)^{-1}\left(\sum_{W_{\lambda} \subseteq V\left(G^{c}\right)}\left(\prod_{i=1}^{r} \frac{(t ; q)_{P_{\lambda_{i}}}}{(q ; q)_{P_{\lambda_{i}}}}\right)\right)
$$

where

$$
P_{\lambda_{i}}= \begin{cases}\left(\left|W_{\lambda_{i}}^{*} \cap E\left(G^{c}\right)\right|+1\right) & \text { if }\left(\left|W_{\lambda_{i}}^{*} \cap E\left(G^{c}\right)\right|+1\right) \leq\left|W_{\lambda_{i}}\right| \\ \left|W_{\lambda_{i}}\right| & \text { otherwise. }\end{cases}
$$

Since $o(G)=o\left(G^{c}\right) \Rightarrow V(G)=V\left(G^{c}\right)$, we have that $W_{\lambda} \subseteq V(G) \equiv W_{\lambda} \subseteq V\left(G^{c}\right)$. Thus, for $1 \leq i \leq r,\left|W_{\lambda_{i}}\right|$ is equal for both $G$ and $G^{c}$ and similarly, $\left|W_{\lambda_{i}}^{*}\right|$ is equal for both $G$ and $G^{c}$. By definition of $G^{c}$,

$$
\left|W_{\lambda_{i}}^{*} \cap E\left(G^{c}\right)\right|=\left(\left(\begin{array}{c}
\left|W_{\lambda_{i}}\right| \\
2
\end{array}\right)-\left|W_{\lambda_{i}}^{*} \cap E(G)\right|\right)=\left(\left|W_{\lambda_{i}}^{*}\right|-\left|W_{\lambda_{i}}^{*} \cap E(G)\right|\right) .
$$


This implies that, with respect to $G^{c}$ and $G, P_{\lambda_{i}}=P_{\lambda_{i}}^{c}$. Therefore, the coeffients (13) and $(14)$ of $m_{\lambda}$ in $Y_{G}^{c}(x ; q, t)$ and $Y_{G^{c}}(x ; q, t)$ are equal.

Using $Y_{G}^{c}(x ; q, t)$, we obtain the following analogues to Propositions $3.1-3.4$ for $G^{c}$.

Proposition 5.1. Let $G$ be a simple graph. $G^{c}$ has order $n$ and size $p$ if and only if the multiplicity of the term

$$
\frac{(t ; q)_{2}(t ; q)^{(n-2)}}{(q ; q)_{2}(q ; q)^{(n-2)}} m_{\left(2,1^{(n-2)}\right)}
$$

is $\frac{2 p}{n(n-1)}$ in $Y_{G}^{c}(x ; q, t)$.

Proposition 5.2. Let $G$ and $H$ be graphs with degree sequences $\operatorname{deg}(G)$ and $\operatorname{deg}(H)$, respectively. Then $o\left(G^{c}\right)=o\left(H^{c}\right)=n, s\left(G^{c}\right)=s\left(H^{c}\right) \leq n$, and $\operatorname{deg}\left(G^{c}\right)=\operatorname{deg}\left(H^{c}\right)$ if and only if the multiplicity of the term

$$
\frac{(t ; q)_{2}(t ; q)^{(n-2)}}{(q ; q)_{2}(q ; q)^{(n-2)}} m_{\left(2,1^{(n-2)}\right)}
$$

is $\leq \frac{2}{(n-1)}$ and is equal in both $Y_{G}^{c}(x ; q, t)$ and $Y_{H}^{c}(x ; q, t)$ and if the coefficients of $m_{((n-1), 1)}$ in $Y_{G}^{c}(x ; q, t)$ and $Y_{H}^{c}(x ; q, t)$ are equal.

Proposition 5.3. Let $G$ be a simple graph of order $n$. Any induced subgraph, $G_{I}^{c}$, of order $(n-1)$ of $G^{c}$ is connected if and only if the multiplicity of the term

$$
\frac{(t ; q)_{(n-1)}(t ; q)}{(q ; q)_{(n-1)}(q ; q)} m_{((n-1), 1)}
$$

in $Y_{G}^{c}(x ; q, t)$ is one.

Proposition 5.4. Let $G$ be a simple graph. $G^{c}$ has order $n$ and is a cycle of size $n$ if and only if the multiplicity of the term

$$
\frac{(t ; q)_{(n-1)}(t ; q)}{(q ; q)_{(n-1)}(q ; q)} m_{((n-1), 1)}
$$

in $Y_{G}^{c}(x ; q, t)$ is one and the multiplicity of the term

$$
\frac{(t ; q)_{2}(t ; q)^{(n-2)}}{(q ; q)_{2}(q ; q)^{(n-2)}} m_{\left(2,1^{(n-2)}\right)}
$$

is $\frac{2}{(n-1)}$. 


\section{References}

[1] B. Bollobas. Graph Theory, An Introductory Course. Springer-Verlag, New York. 1979.

[2] G. Gasper and M. Rahman. Basic Hypergeometric Series. Cambridge University Press, Cambridge. 1990.

[3] N. Jing and T. Jozefiak. A Formula for Two-Row Macdonald Functions. Duke Math. 67, vol.2. 1992. 377-385.

[4] I.G. Macdonald. Symmetric Functions and Hall Polynomials. second edition, Oxford University Press, Oxford. 1995.

[5] B. Sagan. The Symmetric Group. second edition. Springer-Verlag, New York. 2001.

[6] R. Stanley. A Syymetric Function Generalization of the Chromatic Polynomial of a Graph. Adv. Math. 111. 1995. 166-194.

[7] R. Stanley. Graph Colorings and Related Symmetric Functions: Ideas and Applications. Discrete Math. 193. 1998. 267-286. 